galaxies (Moran, op. cit. and Claussen, M.J. et al. Nature 310, 298; 1984) and we could use the statistical parallax technique if we could measure their internal motions with an accuracy of a few microarc seconds per year.

With QUASAT, it might also be possible to determine the orbital motions of maser sources around a galaxy. These angular motions (corresponding to velocities of a few hundred $\mathrm{km} \mathrm{s}^{-1}$ ) will be an order of magnitude larger than the internal motions in the masers themselves. If the rotation curve and the inclination of the galaxy are known, then a distance estimate can be derived directly.

One debate at the workshop centred on the scientific trade-offs between raw resolution and imaging capability. A vocal minority took the view that much cruder images, but with two or three times greater resolution, might lead to more fundamental discoveries. Where to draw the line between resolution and image quality is a matter of judgement but clearly more computer simulations are needed to investigate the point at which image quality deteriorates to levels unacceptable to all.

R.Z. Sagdeev (Space Research Institute, Moscow) gave a brief, but up-to-theminute account of current Soviet thinking. The USSR hopes to place a large $(30 \mathrm{~m})$ antenna in low Earth orbit by the end of this decade. This spacecraft, working with ground telescopes in the USSR, would produce a well-filled aperture but with maximum baselines little longer than can be obtained with current VLBI networks. Thus it is being regarded as a step towards a more ambitious mission which might, like QUASAT, be launched in the 1990s. This second mission resembles QUASAT in several ways: for example, it would employ wider bandwidths and higher observing frequencies. It also offers an appealing way around the trade-off between resolution and image quality which inevitably arise when only one orbiting telescope is used. There would be much to be gained if QUASAT and the Soviet telescope (unofficially dubbed KBACAT) were to be placed in complementary orbits. In the most entertaining few minutes of the entire meeting J.F. Jordan (Jet Propulsion Laboratory) showed a time-lapse movie simulation which demonstrated graphically how, in this regard, the QUASAT-KBACAT baselines would enable us both to have our cake and to eat it, for both longer baselines and a better filled aperture plan are then possible.

QUASAT seems destined to be a competitor in the 1987 selection round in ESA and could get a 'new start' in NASA that same year. The antenna concept is expected to be selected in summer 1985, with a joint NASA-ESA phase A study in 1986. Launch could be in 1992.

P.N. Wilkinson is at the Nuffield Radio Astronomy Laboratories, Jodrell Bank, Macclesfield, Cheshire SKII 9DL, UK.

\title{
Martin Ryle 1918-1984
}

SIR Martin Ryle, pioneer of radio astronomy and explorer of the Universe, died on 14 October 1984. His insight and originality in radio science was first displayed in wartime, when he was recruited directly from Oxford in 1939 to work in the Telecommunications Research Establishment. Here his first tasks were to develop airborne radar aerial systems and to test equipment for the new centimetric waveband; these provided an introduction to radio techniques which prepared him well for his vital, but almost unrecorded, contributions to radio countermeasures.

It was Jack Ratcliffe who persuaded Ryle to start radio research in the Cavendish in 1945, with the support of an ICI Fellowship. The wartime discoveries by John Hey, of intense radio emissions from the Sun and from a mysterious source in the constellation of Cygnus, provided challenging subjects, well suited to Ryle's experience in the location of radio transmitters and the recognition of unusual signal characteristics. He devised a radio interferometer, analogous to Michelson's optical counterpart and soon named after it, which showed in 1946 that sunspots emit powerful, circularly polarized radio waves, and that the quiet sun is surrounded by a hot, radio-emitting corona. A succession of students was encouraged to map this coronal emission, using ingenious developments of the interferometer; these developments can now be seen as the first stages of the technique of aperture synthesis, one of Ryle's most famous achievements.

The investigation of the Cygnus radio source followed similar lines. It was a natural hypothesis that this source might be a star that radiated like the Sun but much more powerfully. The successful location of the source, and its identification as a very distant and very unusual galaxy, opened the way for radio astronomy to investigate the structure of the Universe on the largest scale. At that time, there could be no clear interpretation of the many discrete radio sources appearing in the early surveys; they were at first supposed to belong to our Galaxy, where they would account for the background radio emission from the Milky Way. Ryle determined to survey and locate a large number of these sources. For this purpose he built a large interferometer array, working at a wavelength of $3.7 \mathrm{~m}$. The results were astonishing: the sky was apparently crowded with sources, with almost no concentration in the Milky Way.

In his 1955 Halley lecture, Ryle showed that most of these radio sources must be extragalactic and so distant that they gave the first clear evidence which could be used to distinguish between cosmological theories. His results and their interpretation were immediately the subject of controversy. Australian observations did not agree with the number counts of the radio sources, while the theorists vigorously defended the sinking ship of the Steady State. The conflict could only be resolved through better observations, which Ryle duly provided by improving the interferometer in successive steps, leading eventually to aperture synthesis.

Aperture synthesis, the simulation of a very large radio telescope by the use of multiple interferometers, was Ryle's supreme invention. It was achieved in 1956, when the technical problems of computation were overcome by the use of the poineering digital computer, EDSAC. Its full development progressed through the 'one-mile' telescope (1964), which used three $18 \mathrm{~m}$ paraboloids, giving an angular resolution better than one arc minute, to the well-known 'five kilometer' telescope (1972), working on shorter wavelengths: this telescope gave, for the first time, an angular resolution of one arc second, similar to that of optical telescopes.

The results, both in the form of number counts and as detailed maps of radio galaxies and quasars, firmly established radio astronomy as the main source of direct observational evidence in cosmology. There were, of course, many by-products, most notably Hewish's discovery of pulsars, which led to Ryle and Hewish sharing a Nobel prize in 1974.

Ryle's originality and determination marked the whole development of Cambridge radio astronomy. $\mathrm{He}$ was a magnetic figure, with the power of inspiring of a deep loyalty and a social cohesion among a long succession of students. He had a deep social conscience, which led him in his later years to an active and public concern about nuclear power and armaments. He will be remembered with affection by everyone who worked with him.

F. Graham Smith

\section{0 years ago}

\section{EARTHOUAKE MEASUREMENT}

IN an article on "Earthquakes" in last week's Nature (p. 608), Dr. H. J. Johnston-Lavis takes exception to the records of earthquake motion which I have published, on the ground of their complexity, and pronounces the Plain of Yedo unsuitable for earthquake observations.

Now this seems to me to be a very eclectic way of treating earthquakes. We can measure earthquakes only where we find them, and I suppose the first qualification in a site for an earthquake observatory is that there should be plenty of earthquakes. The Plain of Yedo possesses this qualification in a very high degree; and if the disturbances which occur in it are of a very much more complex character than our $a$ priori notions about earthquakes may have led us to expect, it is not the Plain of Yedo that is to blame.

University College, Dundee. J. A. Ewing

From Nature, 31, 4; 6 November 1884. 